\title{
SACROILIAC JOINT PAIN- PRESENTING AS SCIATICA- A COMMON DIFFERENTIAL DIAGNOSIS
}

\author{
Asha Satish Barge ${ }^{1}$, Satish Mahadeo Barge ${ }^{2}$, Siddhesh Satish Barge ${ }^{3}$
}

${ }_{1}$ MBBS, Department of Pain Management, Barge Pain Clinic, Satara, Maharashtra, India. 2MBBS, Department of Orthopaedics, Mangalmurti Nursing Home, Satara, Maharashtra, India. ${ }^{32}{ }^{\text {nd }}$ Year MBBS, Topiwala National Medical College, Mumbai, Maharashtra, India.

HOW TO CITE THIS ARTICLE: Barge AS, Barge SM, Barge SS. Sacroiliac joint pain- presenting as sciatica- a common differential diagnosis. J. Evolution Med. Dent. Sci. 2019;8(12):953-955, DOI: 10.14260/jemds/2019/211

\section{PRESENTATION OF CASE}

A 72-year-old elderly male, 5 feet 7 inches in height, thin built, weighing $58 \mathrm{~kg}$, farmer by occupation came to our pain clinic for chronic and severe left leg pain along with mild to moderate low back pain for past two and half years. Patient gives history of fall on buttock in March 2013. Patient was operated for a herniated disc at L4-L5 in 2015. After surgery pain did not subside. After trying number of conservative modalities for pain management (physical therapy, analgesics and adjuvant medicines), patient was referred to a psychiatrist. Signs of psychopathology were absent. Then patient was referred to our pain clinic. Leg pain was more than back pain. Pain started in the low back on the left side and referred to left buttock then to the back and along the side of the left leg, ankle and lateral side of the left foot. Pain intensity was 8 out of 10 on numerical rating scale (NRS). Pain and discomfort were sharp, continuous and severely disturbing his sleep pattern. Quality of life was affected significantly. Pain was aggravated on sitting for long, standing, and change of position from sitting to standing. Pain increases on walking and patient feels heaviness as well as weakness of the leg. Patient prefers to sit for few minutes to take rest and then can walk again. Low back pain is mostly on the left side and increases on sitting especially crossed leg within 4-5 minutes. He gets pain on change of position from sitting to standing. Pain also increases significantly on climbing the stairs and prolonged standing. Pain is relieved partially with lying down. Pain is maximum in the morning hours and feels better as the time passes. There is no history of trauma, infection or fever. No Red Flag sign seen. There was no history of any other comorbidities or medical illness. Treatment was already started by the psychiatristPregabalin $75 \mathrm{mg}$ once daily, combination of Tramadol Hydrochloride and Acetaminophen tablet twice daily, Tablet Amitriptyline $10 \mathrm{mg}$ once daily at night. But there was no relief at all despite of continued treatment, so patient was referred to our pain clinic.

Patient was very uncomfortable, depressed, in severe pain and agony. Bowel and bladder functions, appetite, cognitive functions were normal. On inspection the posture was abnormal with antalgic gait affecting left leg indicating pain with weight bearing on left side. Spine and pelvic asymmetry were noted. Kyphoscoliosis was seen. Range of motion of left hip was affected moderately.

'Financial or Other Competing Interest': None.

Submission 28-09-2018, Peer Review 03-11-2018,

Acceptance 10-11-2018, Published 25-03-2019.

Corresponding Author:

Dr. Asha Satish Barge,

MBBS, Department of Pain Management,

Barge Pain Clinic, Satara, Maharashtra, India.

E-mail:bargeasha368@gmail.com

DOI: $10.14260 /$ jemds $/ 2019 / 211$
On palpation, area lateral to the left ischial tuberosity was very tender suggesting the cause was located in the gluteal area itself. SLR (straight leg raising) test was positive and significantly affected at 50 degree. Bragard test was negative but Kemp sign was positive.

The general examination, respiratory, cardiovascular and gastrointestinal systems revealed no positive findings. CNS examination showed normal higher motor functions and normal cranial nerves. Sensory examination revealed paraesthesia in left leg in a non-dermatomal pattern. Motor system examination was normal showing adequate strength of the muscles of the lower extremity.

Sacroiliac joint provocation tests were done. Thigh thrust, the distraction test, sacral thrust, FABER test (Flexion, abduction and external rotation of hip), were positive.

Diagnosis was based on detailed clinical history and examination.

\section{CLINICAL DIAGNOSIS}

Sacroiliac Joint Arthropathy Presenting as Sciatica

\section{DIFFERENTIAL DIAGNOSIS}

1. Prolapsed Intervertebral Disc

2. Pyriformis Syndrome

3. Lateral Canal Stenosis

\section{PATHOLOGICAL DISCUSSION}

Routine blood investigations, ESR, fasting blood sugar, BT, CT, PT, INR were normal. RA test was negative.

Plain x-ray lumbosacral spine showed kyphoscoliosis and extensive sclerosis with degenerative changes especially in lumbar region.

MRI scan was done which showed herniated disc and scar tissue.

After obtaining informed consent, under all aseptic precautions, we performed diagnostic come therapeutic left sided sacroiliac joint injection under fluoroscopy which gave significant pain relief to the patient, and also our diagnosis was confirmed. Sacroiliac joint RF denervation was advised if needed.

Sciatica with low back pain and leg pain is very common finding in our clinical practice. The purpose of this case report is to differentiate between various causes of Low back pain and leg pain and overcome the diagnostic dilemma.

There are many different conditions which can cause Low back pain and leg pain. These conditions can be divided in two different categories, patient with Predominant Back pain and Patient with Predominant leg pain. Our patient presented with predominant leg pain on left side. The associated Low back pain can have multiple differential diagnosis such as Radiculopathy, Prolapsed disc, lateral canal stenosis, Sacroiliac joint dysfunction, myofascial pain syndrome, piriformis syndrome and so on. 
Testing of sensory and motor function revealed no abnormality. EMG/ NCV Studies not performed. Though EMG/ NCV studies are very useful in cases of Radiculopathy.

Sciatica is a common syndrome in clinical practice and mostly considered to be because of root compression due to disc herniation, lateral or foraminal stenosis, spondylolisthesis, or tumour. However, in Our case report it is very clear that other mechanisms may prevail and referred pain from Sacroiliac joint can mimic sciatica like symptoms.

Root compression itself is not always a cause for sciatica. Thus, root compression with or without inflammation is just one of the causes of radicular pain or sciatica.

Diagnosis of root compression is probable, when on physical examination, paresis, sensory loss, reflex changes which are the neurological signs; or a positive SLR or Bragard test are found. However, with our clinical judgement, in many patients of sciatica which come to the pain clinic or to a specialized neurological clinic; often no underlying Lumbar disc abnormality can be found. If there are no neurological signs or symptoms in patients of sciatica or radicular pain, other sources of pain such as sacroiliac joint should be considered as a pain generator. Sacroiliac joint related pain shows pattern similar to L5-S1 dermatomal pattern. It can refer to the buttocks, thigh, upper and lower leg to the lateral ankle. In these patients SLR test can be positive with pain in the sacroiliac area. Sacroiliac joint pain is very difficult to diagnose on physical examination. The diagnosis is based on a pattern of findings, no single SI joint test is validated and a large variation in reliability is reported. $(1,2)$ In clinical practice; few of asymptomatic patients, these tests can be positive. In patients with SI joint related pain, Pain referral maps are of limited value.

There are no specific radiological abnormalities for this syndrome, although CT scan and MRI can be helpful in diagnosing sacroiliitis and joint space narrowing. At least MRI spine should be advocated before a definitive diagnosis of SI joint related pain is made. SIJ dysfunction can occur in patients with low back pain and sciatica and imaging- proven disc herniation. Thus, when treatment is focused on the presence of SIJ dysfunction spine surgery can be prevented in these patients. To make a clinical diagnosis, the International Association for the study of Pain (IASP) has proposed a set of criteria for diagnosing SIJ pain. According to these criteria, SIJ pain refers to patients with pain in the area of the SI joint, which should be reproducible by performing specific provocation SIJ tests or should be completely relieved by infiltration of SIJ with local anaesthetic agents. With clinical suspicion of a SI joint origin of pain intra- articular injection is currently the only means to confirm that diagnosis, although its validity is questioned. (3) If SI joint pain is confirmed, it can be treated by injection with corticosteroids or by radiofrequency ablation. (4)

Buttock and lower extremity pain can be ablated by the introduction of local anaesthetic into the joint space under image intensifier guidance and pain referral maps in symptomatic patients are available. These facts provide a strong case for the SIJ as a potential and sole source of pain in specific patients with buttock and lower extremity pain. (5)

Apart from root compression and the SI joint, diseases of the lumbar facet joint and spinal tumours may cause radiating pain into the leg. If imaging techniques do not reveal a possible cause of pain, differentiation between these possible sources should be performed by blockades with local anaesthetics, although false positive findings and low specificity and sensitivity have been reported. (6)

Explanation for the radiating pain pattern into the leg due to SI joint are many. It can be a referred pain from this joint, just like visceral pain. The radiating pain pattern depends on the segmental nerve supply of SI Joint (L5 - S4).

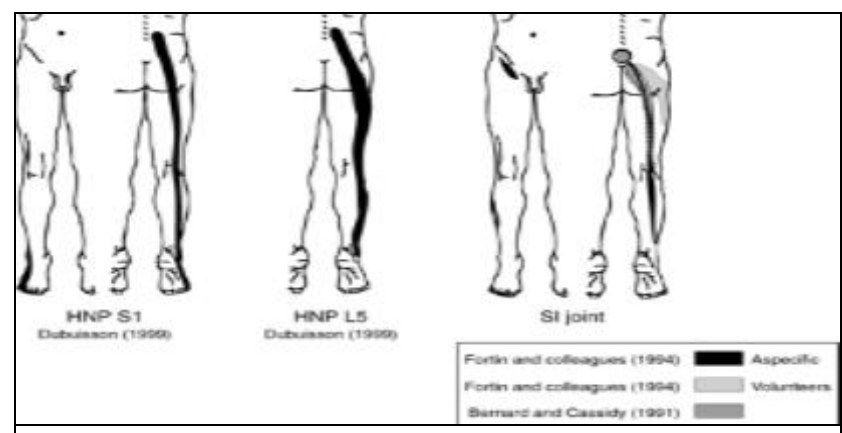

Figure 1. Schematic Representation of Radiating Pain Due to Root Compression L5 or S1, or Due to Disease of SI Joint

Finally, one should take into account that radiating pain into the leg can also be derived from other structures in the lower back; other than the SI joint, particularly when they are supplied by branches from the same spinal nerves, that is L5S4.

The centralization phenomenon has been repeatedly described and evaluated for reliability and validity. $(7,8,9,10)$

Subsequently it has been found to be highly specific to discogenic pain and is not observed in patients with confirmed SIJ pain or facet joint pain. $(11,12,13)$ On this basis it seems reasonable to assume that SIJ tests, positive in the presence of the centralisation phenomenon, are falsely positive.

Thus, diagnosis is made by exclusion of the more common causes first. Careful examination of gait, posture and alignment is must. Detailed examination of hip, pelvis and SI joint along with lumbar spine is mandatory so as to find out the primary pain generator.

No single finding is reliable in all cases, but one can get a reasonable clinical picture with careful clinical examination. SLR test is not very specific.

Radiological investigations have limited role.

Differential diagnosis includes Low back pain, Radiculopathy, Facet syndrome, SI joint disease, MPS, Conditions irritating the sciatic nerve, Trochanteric bursitis, Pelvic tumour, Endometriosis.

\section{DISCUSSION OF MANAGEMENT}

Intra-articular injection of SI joint with local anaesthetic is considered as the gold standard for diagnosing SI joint related pain and should be performed under fluoroscopy or CT guidance.

If SI joint pain is confirmed, it can be treated by injection with corticosteroids or by RF denervation.

Sciatica is a common syndrome which can have various causes. In absence of neurological signs mostly related to root compression with or without inflammation, other sources for sciatica should be ruled out. Many cases of SI joint disease can present with sciatica. Single SI joint test is not reliable. Three or more SI provocation tests are helpful to come to a clinical diagnosis. The gold standard for establishing a definitive 
diagnosis of SI joint related pain is intra- articular low volume local anaesthetic injection. One should always keep in mind that sciatica can be a referred pain from SI joint, which will avoid many unnecessary investigations as well as surgeries. Moreover, patients can successfully be treated for this condition.

\section{FINAL DIAGNOSIS}

Sacroiliac Joint Arthropathy Presenting as Sciatica

\section{REFERENCES}

[1] Van der Wurff P, Buijs EJ, Groen GJ. A multitest regimen of pain provocation tests as an aid to reduce unnecessary minimally invasive sacroiliac joint procedures. Arch Phys Med Rehabil 2006;87(1):10-4.

[2] Van der Wurff P, Buijs EJ, Groen GJ. Intensity mapping of pain referral areas in sacroiliac joint pain patients, J Manipulative Physiol Ther 2006;29(3):190-5.

[3] Berthelot JM, Labat JJ, Le Goff B, et al. Provocative sacroiliac joint maneuvers and sacroiliac joint block are unreliable for diagnosing sacroiliac joint pain. Joint Bone Spine 2006;73(1):17-23.

[4] Cohen SP. Sacroiliac joint pain: a comprehensive review of anatomy, diagnosis, and treatment. Anesth Analg 2005;101(5):1440-53.

[5] Forst SL, Wheeler MT, Fortin JD, et al. The sacroiliac joint: anatomy, physiology and clinical significance. Pain Physician 2006:9(1):61-7.

[6] Wolff AP, Groen GJ, Wilder-Smith $\mathrm{OH}$. Influence of needle position on lumbar segmental nerve root block selectivity. Reg Anesth Pain Med 2006;31(6):523-30.
[7] Clare HA, Adams R, Maher CG. Reliability of McKenzie classification of patients with cervical or lumbar pain. J Manipulative Physiol Ther 2005;28(2):122-7.

[8] Donelson R. Rapidly reversible low back pain: an evidence based pathway to widespread recoveries and savings. Hanover, NH: SelfCare First, LLC 2007: p. 233.

[9] Werneke MW, Hart DL. Centralization: association between repeated end-range pain responses and behavioural signs in patients with acute non-specific low back pain. J Rehabil Med 2005;37(5):286-90.

[10] Werneke M, May S. The centralization phenomenon and fear - avoidance beliefs as prognostic factors for acute low back pain. J Orthop Sports Phys Ther 2005;35(12):844-5.

[11] Laslett M, Oberg B, Aprill CN, et al. Centralisation as a predictor of provocation discography results in chronic low back pain, and the influence of disability and distress on diagnostic power. Spine J 2005;5(4):370-80.

[12] Laslett M, Aprill CN, McDonald B, et al. Clinical predictors of lumbar provocation discography: a study of clinical predictors of lumbar provocation discography. Eur Spine J 2006;15(10):1473-84.

[13] Laslett M, McDonald B, Aprill CN, et al. Clinical predictors of screening lumbar zygapophysial joint blocks: development of clinical prediction rules. Spine J 2006;6(4):370-9. 